Document downloaded from:

http://hdl.handle.net/10251/65355

This paper must be cited as:

Gonzalez Iglesias, D.; Monerris Belda, O.; Diaz, M.; Gimeno Martinez, B.; Boria Esbert, VE.; Raboso García-Baquero, D. (2015). Experimental Analysis of the Multipactor Effect With RF Pulsed Signals. IEEE Electron Device Letters. 36(10):1085-1087. doi:10.1109/LED.2015.2468068.

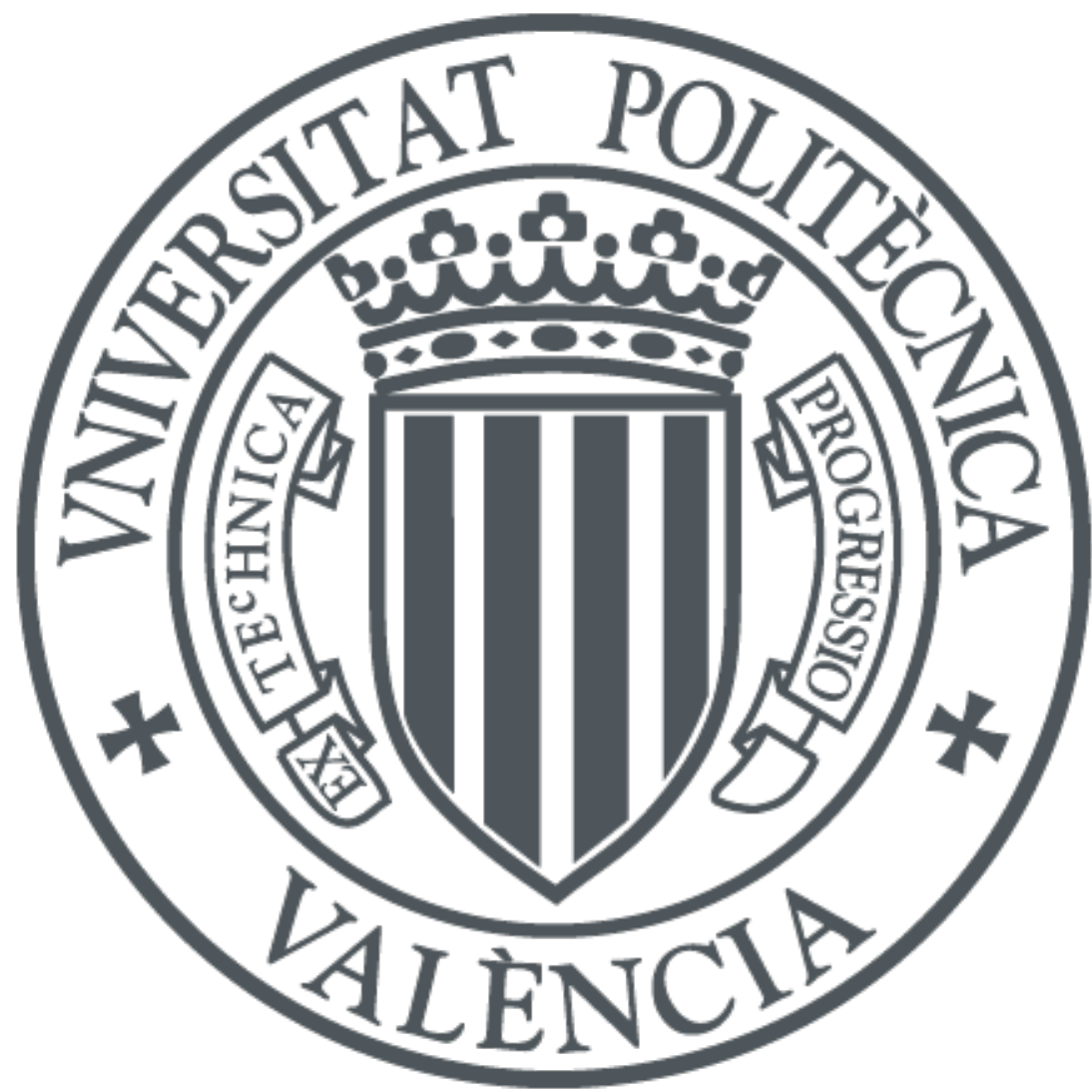

The final publication is available at

http://dx.doi.org/10.1109/LED.2015.2468068

Copyright Institute of Electrical and Electronics Engineers (IEEE)

Additional Information

(C) 2015 IEEE. Personal use of this material is permitted. Permission from IEEE must be obtained for all other uses, in any current or future media, including reprinting/republishing this material for advertising or promotional purposes, creating new collective works, for resale or redistribution to servers or lists, or reuse of any copyrighted component of this work in other works. 


\title{
Experimental Analysis of the Multipactor Effect with RF Pulsed Signals
}

\author{
D. González-Iglesias, O. Monerris Belda, M. E. Díaz, B. Gimeno, Member, IEEE, \\ V. E. Boria, Senior Member, IEEE, D. Raboso
}

\begin{abstract}
The main goal of this work is the analysis of the multipactor effect within a coaxial waveguide excited by an RF pulsed signal. The variation of the multipactor RF voltage threshold with the $\mathrm{ON}$ interval length of the pulse has been analyzed. To reach this aim, an in-house multipactor simulation code based on the Monte-Carlo algorithm has been implemented. Numerical simulations show that the multipactor RF voltage threshold increases as the $\mathrm{ON}$ pulse interval diminishes. In addition, an experiment was carried out to validate the proposed theoretical model, demonstrating the excellent agreement between theory and experimental data. Finally, the results are compared to the "20-gap-crossing" rule used in the space standard document (ECSS-E20-1A).
\end{abstract}

Index Terms-Multipactor effect, RF breakdown, coaxial waveguide, RF pulse, "20-gap-crossing” rule.

\section{INTRODUCTION}

Multipactor breakdown is an electron avalanche-like discharge occurring in components operating under vacuum conditions and high-power RF electromagnetic fields [1]. It can appear in a wide variety of scenarios, such as satellite passive communication devices, klystrons, and accelerator structures. The multipactor effect occurs when an RF electric field accelerates free electrons inside the device, impacting on the metallic walls of the structure. If the kinetic energy of the impacting electrons is high enough, secondary electrons may be released from the surface thus increasing the electron population inside the device. Once the number of electrons becomes very high, an electrical current is established between the walls of the component, degrading the component performance.

Focusing on space communications, multipactor reveals as a restrictive limitation to the power handling capabilities of many satellite RF and microwave devices. This is quite an inconvenience since the increasing number of users, requiring higher and higher bit rates, implies the necessity of increasing power levels in the RF and microwave payload equipment.

Multipactor has been deeply studied in many kinds of geometries, involving different surface materials (metals, dielectrics and ferrites). Most of this work has been made

D. González-Iglesias and B. Gimeno are with Depto. Física AplicadaICMUV, Universidad de Valencia, Spain.

O. Monerris Belda is with the Val Space Consortium, Valencia, Spain.

M. E. Díaz is with Depto. de Informática, ETSE, Universidad de Valencia, Spain.

V. E. Boria is with Depto. Comunicaciones-iTEAM, Universidad Politécnica de Valencia, Spain.

D. Raboso is with European Space Agency, ESA/ESTEC, The Netherlands.

Our acknowledgement to Val Space Consortium for its contribution (Laboratories funded by the European Regional Development Fund) A way of making Europe. assuming a time harmonic excitation, i.e. continuous wave (CW) operation. However, in many microwave applications, pulsed operation involving pulses of finite length in time separated by zero power intervals is employed rather than the CW case. Even more, in telecommunication satellites, within the pulses, multicarrier operation with one or more modulated carriers is employed in order to use efficiently the limit frequency band available. Up to now, the standard adopted by the European Space Agency (ESA) for multicarrier multipactor design is based on the "20-gap-crossing" rule [2], which is used as an engineering "rule of thumb" for assessing the risk of multipactor breakdown. Understanding of the multipactor phenomenon when involving constant envelope RF pulses, seems to be the first step towards the comprehension of realistic signals, where the signal envelope varies with time within the pulse. According to the authors' knowledge, there are few studies concerning microwave pulse width effect in the multipactor discharge [3]-[5], but none of them present both theoretical and experimental results. In this manuscript, we analyze the multipactor behavior in constant envelope RF pulses from both a theoretical and experimental point of view. The numerical simulations carried out with our developed in-house multipactor code present a good match with the experimental data. Thus, it is both theoretically and experimentally demonstrated the inefficacy of the "20-gapcrossing" rule predictions and it is revealed the need of more realistic rules for the design of components operating under multicarrier regimes.

This letter analyses the effect of the ON pulse duration in the multipactor RF voltage threshold within a standard coaxial transmission line. During the ON interval of the RF pulse, the electron population in the device grows whenever the RF voltage exceeds the $\mathrm{CW}$ multipactor $\mathrm{RF}$ voltage threshold. However, during the OFF interval the electron population drops, since the electrons are mainly absorbed by the walls of the component. For the onset of a multipactor discharge a high growth of the initial electron population is required and, if the ON pulse interval is short enough, the electron population might not reach the minimum required to trigger the discharge. In this work, we focus on the analysis of low duty cycle pulses, i. e. the time elapsed between two consecutive ON intervals is long enough to ensure electron population decays to zero between consecutive pulses.

The document is organized as follows. In Section II, the theoretical model employed for multipactor simulations is discussed. Next, in Section III, numerical and experimental results are shown for a particular coaxial sample. A brief 
explanation of the multipactor experimental set-up is also included. Besides, the results are compared to the multipactor RF voltage thresholds derived from the "20-gap-crossing" rule. Finally, in Section IV, the conclusions are outlined.

\section{THEORY}

To analyse the multipactor phenomenon under the excitation of pulsed and ultra short pulsed signals, a home-made software based on the individual electron model has been implemented for coaxial transmission lines. This code is based on the 3-D tracking of a set of electrons. Each electron is individually tracked. Electron motion is governed by the RF electric and magnetic fields corresponding to the fundamental coaxial TEM mode. Equation of the electron motion is derived from Lorentz force, and is solved numerically by means of the VelocityVerlet algorithm [6]. When an electron collides with the metallic coaxial walls, the impacting electron can be absorbed, it can be elastically or inelastically backscattered, or true secondaries can be produced. Each of these possible interactions between the electron and the surface are modeled as the different contributions of the Secondary Electron Yield (SEY) function. In this work, the SEY model and formulas described in [7], [8], which were already successfully implemented in CST Particle Studio [9], are employed.

The multipactor onset criteria is based on the detection of a high enough electron population within the component, in order to ensure that the discharge effects are noticeable from an experimental point of view. According to the multipactor literature, the electron population is expected to grow until a steady-state (saturation level) is reached [10]. At this stage the electron population is maximum (with oscillations around this value) and the detection is supposed to take place. Previous numerical simulations presented in [11] show that the saturation level is within the range of $10^{9}-10^{12}$ electrons, depending on the particular characteristics of the case (waveguide dimensions, SEY properties, RF voltage). In this work we chose the saturation level of $N_{\text {sat }}=10^{12}$ since, after trying different levels of $N_{s a t}$, we saw that the aforementioned value is the best one to fit the experimental data. This value has been set as the population level to determine the onset of the multipactor discharge in the simulation routine.

\section{NUMERICAL AND EXPERIMENTAL RESULTS}

The coaxial dimensions for multipactor simulations, as well as for the test sample, are $a=1.238 \mathrm{~mm}$ and $b=2.850 \mathrm{~mm}$ for the inner and outer conductor radii, respectively; thus, the gap length is $d=b-a=1.612 \mathrm{~mm}$. The coaxial length is $100 \mathrm{~mm}$. Both conductors are made of copper and the SEY data for this material is extracted from [12]. According to the model proposed in [8], the fitting parameters for the SEY true secondaries contribution are: $\delta_{m}=1.77, W_{m}=277 \mathrm{eV}$, and $s=1.539$. The operational test frequency is $f=1.145 \mathrm{GHz}$.

The starting number of electrons in the simulations is $N_{i}=500$, and the final number of electrons at which the code stops is $N_{e}=10^{4}$. As stated in the previous section, the saturation level for the onset of the discharge is chosen to be $N_{\text {sat }}=10^{12}$. However, due to computational

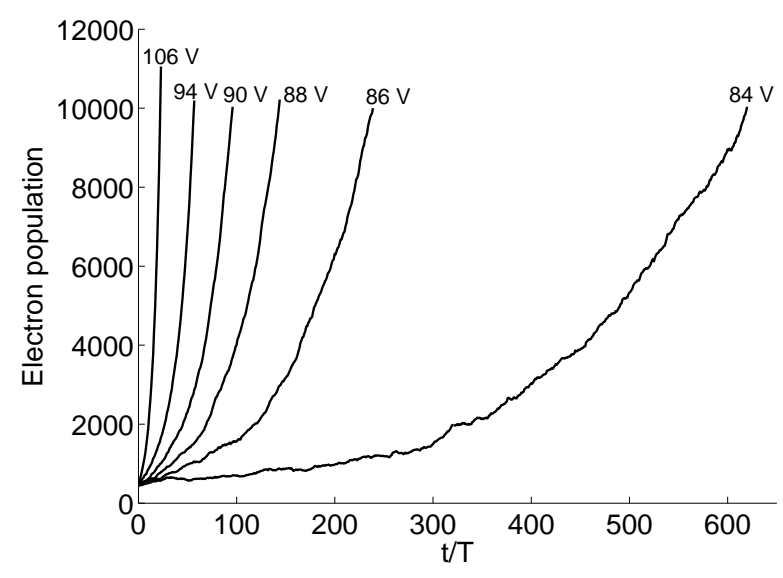

Fig. 1. Evolution of the electron population within the coaxial waveguide excited by a continuous wave signal as a function of the time normalized to the RF period for different RF voltages $(T=1 / f)$.

limitations, a standard PC is not able to hold a simulation where such a number of particles is simultaneously tracked. In fact, other existing simulation codes use the called superparticles that contain a number of electrons (even fractions of them), all with the same dynamical variables. Nevertheless, using this approach, the statistical representation of the process becomes worse as the number of electrons increases while the number of super-particles becomes constant. To overcome this inconvenience, the following approach is proposed relying on the numerical results obtained from the simulations with $N_{e}=10^{4}$ being the maximum number of simulated electrons. The electron population as a function of the RF voltage is depicted in Fig. 1. These data show that the electron population increment follows an exponential trend. For a pure continuous wave RF signal, the presence of this exponential trend in the electron population would be sufficient to ensure that the discharge will eventually occur. However, this criterion is not enough in this case, since we are dealing with pulsed signals where the RF wave is active only during the time $t_{o n}$. Numerical results shown in Fig. 1 for different amplitudes of the RF voltage are fitted to an exponential function, and then are used to predict the time lapse that requires the electron population to reach the saturation level. If the predicted time lapse is lower than $t_{o n}$, it is assumed that multipator can take place. This method can be used to estimate the multipactor RF voltage threshold for any length of the RF pulse.

In order to validate the theoretical model, an experimental test campaign was carried out at the ESA-VSC [13] European high power radiofrequency joint laboratory; the experimental set-up (see Fig. 2) is the standard one commonly employed in multipactor measurements (see [2] for a detailed description). Three detection methods were employed: the measurement of nulling between the incident and the reflected waves at the carrier frequency, the third-harmonic frequency detection of the transmitted signal, and the measurement of the secondary electrons generated in the discharge with a biased electron probe. Following the multipactor testing ECSS standard [2], in order to determine the threshold level it is required a minimum of two detection methods simultaneously triggered. 


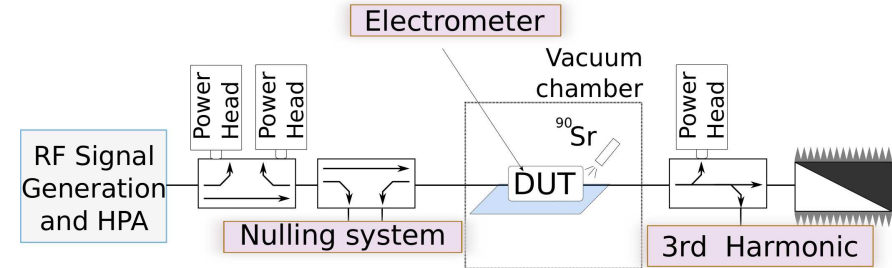

Fig. 2. Multipactor test bed diagram with the detection systems. Note that a Strontium-90 radioactive source was employed for electron seeding purpose.

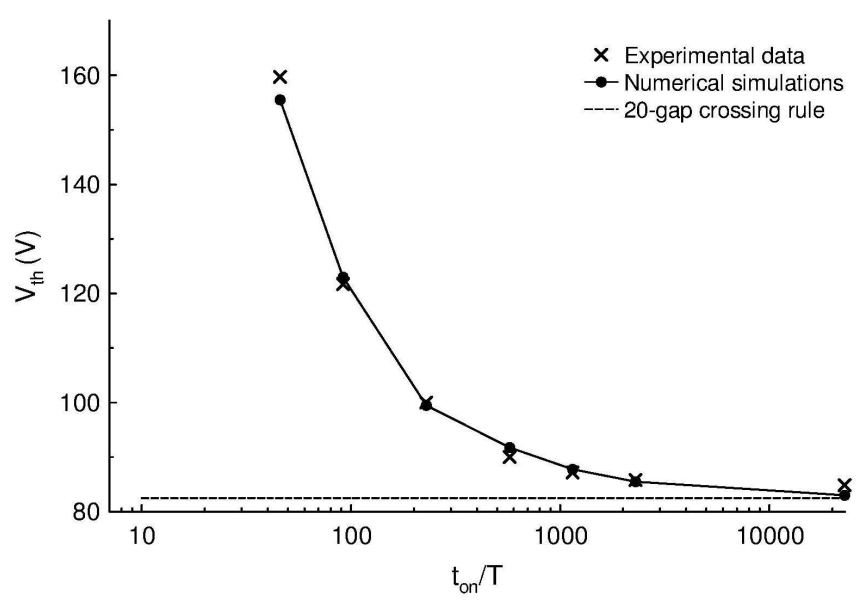

Fig. 3. Multipactor RF voltage threshold for several RF pulse lengths normalized to the RF period of the carrier signal. Experimental and theoretical results, as well as the 20 -gap-crossing rule predictions, are shown.

From the experimental point of view, it was observed that for pulses longer than $1 \mu$ s all the detection system were monitored together. Nevertheless, for shorter pulses it was observed that only the electrometer and the harmonic system were able to detect the discharges.

Both experimental and theoretical results are shown in Fig. 3 showing good agreement between them. It is noticed that the shorter the pulse, the higher the RF multipactor voltage threshold. In Fig. 1 it was already noticed that higher $\mathrm{RF}$ voltage involves higher rate of growth in the electron population. Consequently, when the pulse length diminishes a faster increase in the electron population is needed to reach the same saturation level. It must also be mentioned that ultra short pulses with $t_{\text {on }} / T=22.9$ and $t_{\text {on }} / T=11.45$ were measured, but no multipactor discharge was detected up to $199 \mathrm{~V}$, which was the maximum RF voltage delivered by the available RF power amplifier. Multipactor simulations for the two aforementioned pulses also predicted no multipactor discharge up to the voltage tested level. Measuring multipactor in pulses shorter than $t_{\text {on }} / T=11.45$ was not possible, since considerable distortion of the RF pulse envelope was observed due to limitations in the RF power amplifier performance.

It must be also mentioned that the experimental reusits shown in this work are consistent with those presented in [5], where the variation of the multipactor RF power threshold with the pulse width was tested. However, pulses shorter than 240
$\mathrm{T}$ were not considered in [5], thus not considering the very interesting region concerning pulses of very short length.

Finally, a brief comparison between our results and the predictions of the "20-gap-crossing" rule [2] is presented. Basically, this rule states that with a multi-carrier signal, multipactor events will be detectable whenever the power is maintained above the $\mathrm{CW}$ threshold for a time period about 20 gap crossings of the electron. For this particular case, $f \times d=1.846 \mathrm{GHzmm}$, so multipactor with order one is expected. Consequently, the time for 20 crossing gaps is $10 \mathrm{~T}$. According to this rule, the multipactor RF voltage threshold is a flat line (see Fig. 3) at the level of the continuous wave threshold for pulses longer than $10 \mathrm{~T}$, whereas no discharge would be expected below this time. It can be concluded that the "20-gap-crossing" rule is not applicable in this case, since it does not reproduce the experimental results.

\section{CONCLusions}

In this letter, the authors have studied the multipactor effect with RF pulsed signals within a coaxial waveguide. An inhouse code was developed to perform multipactor simulations, and the results obtained from the experimental test are in excellent agreement to the theoretical predictions. Both numerical and experimental results show the increment in the multipactor RF voltage threshold as the ON pulse interval shortens. To conclude, it has been shown that the so-called "20-gap-crossing" rule reveals considerable discrepancies with the results presented in this work.

\section{REFERENCES}

[1] J. Vaughan, "Multipactor", IEEE Trans. Electron Devices, vol. 35, no. 7, pp. 1172-1180, July 1988 .

[2] "Multipaction design and test", ECSS-E-20-01A, ESA-ESTEC, 2003.

[3] J. Rasch, D. Anderson, and V. E. Semenov, "Multipactor breakdown in microwave pulses", Journal of Physics D: Applied Physics, vol. 46, no. 50, 505201 (2013).

[4] S. Anza, C. Vicente, B. Gimeno, V. E. Boria, J. Armendáriz, "Long-term multipactor discharge in multicarrier systems", Physics of Plasmas, vol. 14, 082112 (2007).

[5] N. Le Gallou, C. Miquel-Espanya, S. Matinfar, C. Ernst, F. De Paolis, "Multipactor threshold and margins using realistic modulated signals", MULCOPIM 2011, 21-23 September 2011, Valencia (Spain).

[6] L. Verlet, "Computer 'experiments' on classical fluids. I. Thermodynamical properties of Lennard-Jones molecules", Phys. Rev., vol. 159, no. 1, pp. 98-103, Jul. 1967.

[7] M. A. Furman and M. T. F. Pivi, "Simulation of secondary electron emission based on a phenomenological probabilistic model", Tech. Rep. LBNL-52807, Jun. 2003.

[8] J. de Lara, F. Pérez, M. Alfonseca, L. Galán, I. Montero, E. Román, D. R. García-Baquero, "Multipactor prediction for on-board spacecraft RF equipment with the MEST software tool", IEEE Transactions on Plasma Science, vol.34, no. 2, pp. 476-484, April 2006.

[9] www.cst.com

[10] S. Riyopoulos, "Multipactor saturation due to space-charge-induced debunching", Physics of plasmas, issue 5, vol. 4, pp. 1448-1462, 1997.

[11] E. Sorolla, A. Sounas, M. Mattes, "Space charge effects for multipactor in coaxial lines", Physics of Plasmas, vol. 22, 033512 (2015).

[12] V. Baglin, I. Collins, B. Henrist, N. Hilleret, G. Vorlaufer, "A summary of main experimental results concerning the secondary emission of copper", LHC Project Report 472, CERN, Geneva, Switzerland, 2002.

[13] Val Space Consortium-European Space Agency RF High-Power Laboratory, Valencia, Spain (www.val-space.com). 